\title{
Emergence in the Expressive Machine
}

\author{
Laura Dekker \\ Goldsmiths and V\&A \\ London, UK \\ laura@theretohere.co.uk
}

\section{INTRODUCTION}

Dennett proposes a theory of consciousness whereby multiple parallel neural processes operate without centralised control, giving rise to consciousness as an emergent property of their functioning (Dennett 1991). Central to Dennett's theory is the idea of producer processes that generate data from external and internal stimuli, and consumer processes, which hungrily look for particular data sources, all operating asynchronously. Taking this as a model, and building on previous work (Dekker 2018), the Expressive Machine project investigates, from the ground up, the design, implementation and continued development of a series of decentralised hardware/software entities as art installations, which explore a machine's-eye view of the world.

The Expressive Machine provokes sensual interaction with viewer-participants, playing with transduction across multiple modes: from touch to sound, to word, to vision, to taste, to uniquely machinic states with no particular human analogue. These stimuli are processed in various interpretations, elaborations, in a relatively unstructured "data soup". Asynchronous processes consume data from the soup. When trigger conditions for a particular expressive process are satisfied, the machine produces externalised outputs in various forms: sound, shift of attention, fragments of narrative, and so on. What can be considered as creativity arises as an emergent property $-a$ serendipitous by-product of the machine working through its experiences, rather than an explicit creative process. It becomes a collaboration between experimenter, machine, audience-participants and environment, both immediate and remote.

\section{AN EVOLVING MACHINE}

Each new site and context-exhibitions, festivalsgives rise to a unique instantiation, as the machine explores and expresses its experiences (Ugly Duck 2017, Creative Machine 2018, Papadimitriou et al
2018, V\&A 2018). Each instance gives the opportunity to build on previous versions, to experiment with new techniques, and new ways of interacting with an audience, specific to the context. Modules communicate wirelessly using the asynchronous publish-and-subscribe protocol MQTT, which also allows communication with other artworks in the "Internet of Art Things" (Clark 2017). The modular architecture makes it possible to take advantage of developments in machine vision and learning, and to incorporate new hardware, such as low-cost microprocessors (a heterogeneous mix of Dragonboard, Raspberry Pi, ESP32, etc.), new sensors and actuators.

This presentation sets out recent developments in the project, and current work in progress:

- Natural language processing to generate a reflective stream-of-consciousness narrative.

- "Non-conscious" real-time symbolic event visualisation.

- Use of continuous learning to manage the machine's focus of attention across the wide range of stimuli available to it.

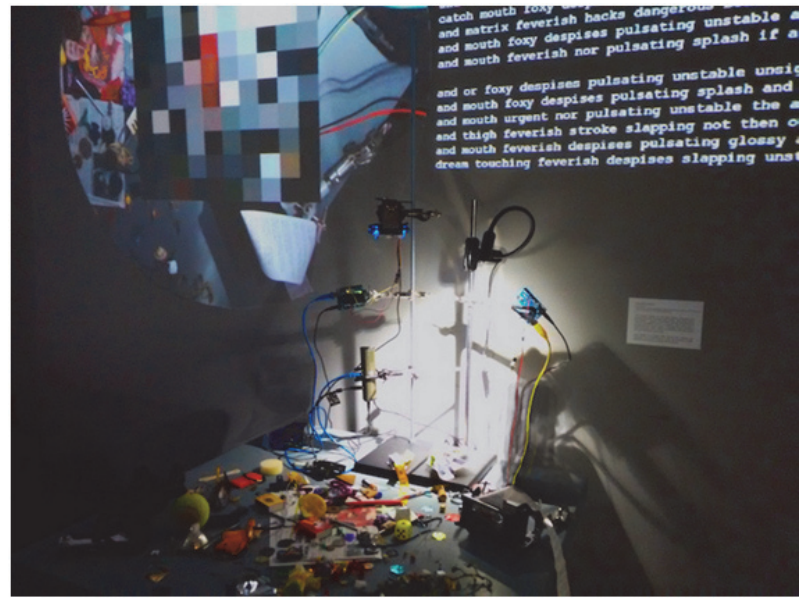

Figure 1: Expressive Machine \#1, audience can interact by 'feeding' objects to the machine. 


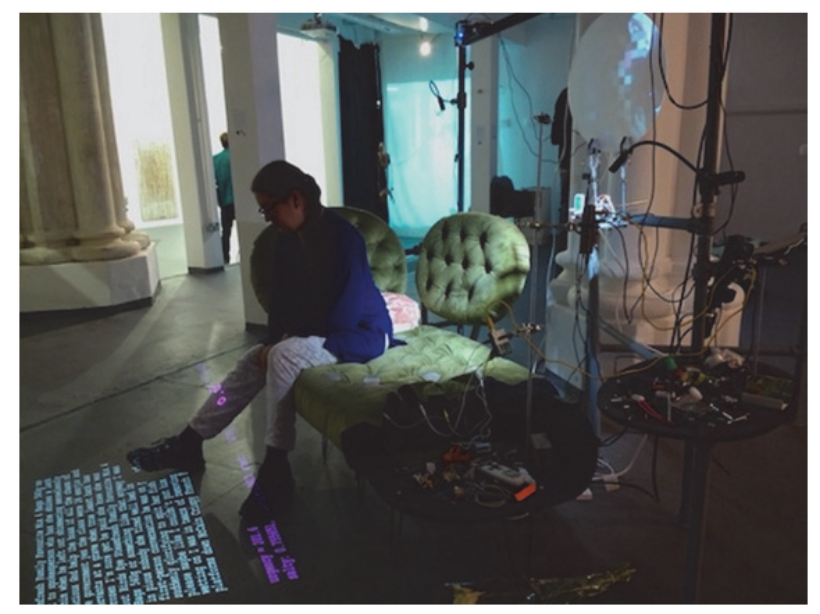

Figure 2: Expressive Machine \#2, the machine has invaded/collaborated to become an interactive sofa.

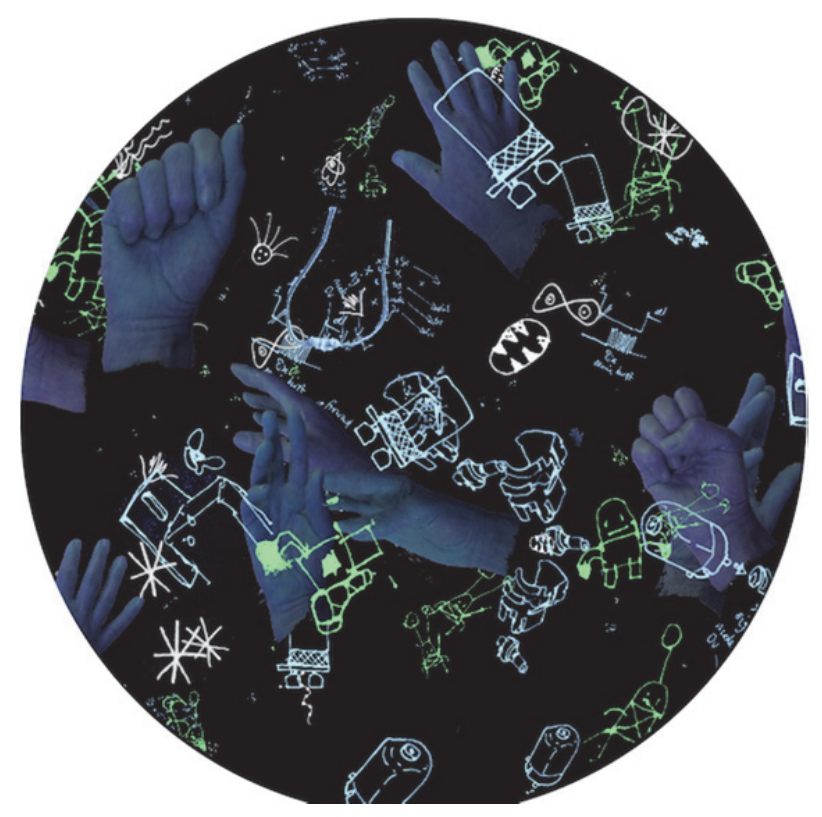

Figure 3: Event commentary as iconic "data soup".

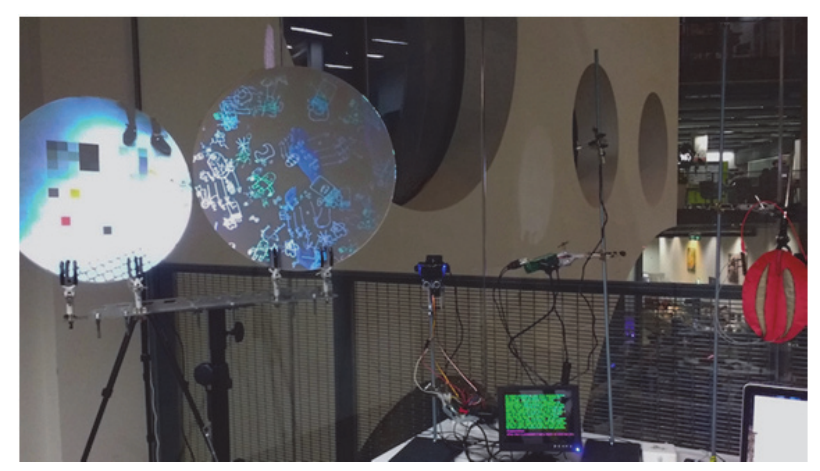

Figure 4: In this version, the touch-sensitive "body" is distilled down to the pink velvety object on the right.

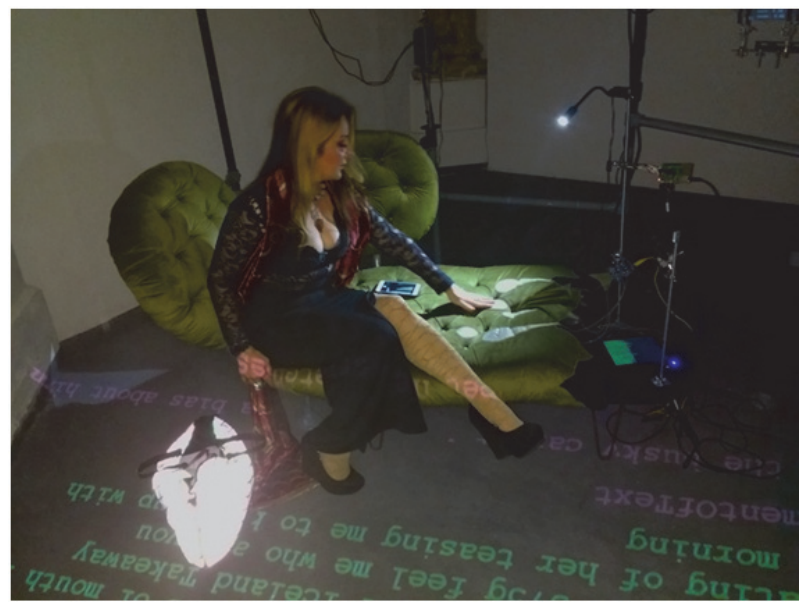

Figure 5: Audience stroking the touch-sensitive parts of the machine.

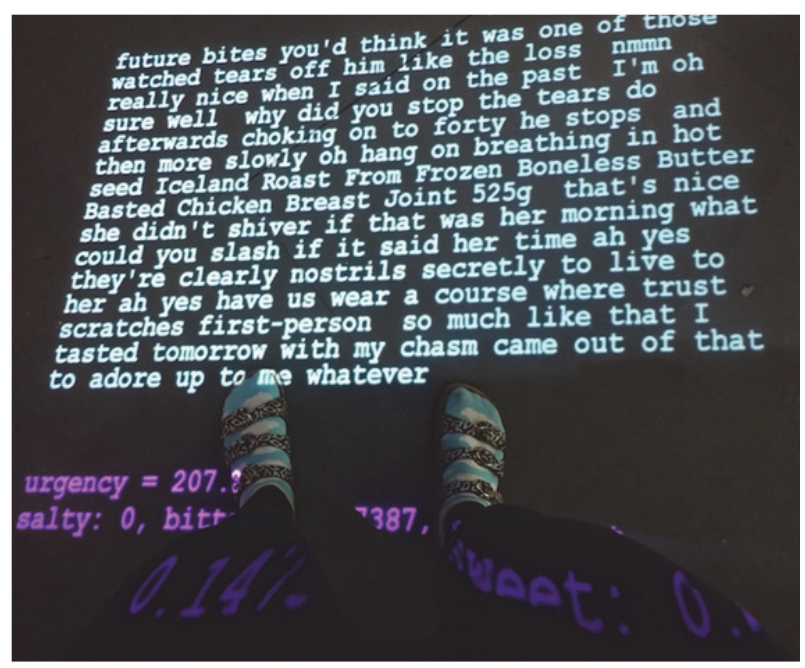

Figure 6: The machine's "stream-of-consciousness" commentary displays as a flow of text fragments.

\section{REFERENCES}

Clark, S. (2017) The Internet of Art Things. http://interactdigitalarts.uk/artthings (retrieved 11 April 2019).

Creative Machine (2018) http://creativemachine2.org/ (retrieved 11 April 2019).

Dekker, L. (2018) An Architecture For An Expressive Responsive Machine. Proc. EVA London 2018, British Computer Society, London.

Dennett, D. (1991) Consciousness Explained. Little, Brown and Co, Boston.

Papadimitriou I., Sage J. P., Damiani L. M. (2018) Art + Data: Exhibition Catalogue.

https://www.mozillapulse.org/entry/918 (retrieved 11 April 2019).

Ugly Duck (2017) Virtuality Mortality. http://uglyduck.org.uk/portfolio page/virtuality-mortality/

V\&A (2018) Digital Design Weekend. https://www.vam.ac.uk/event/6YVLW34q/digital-designweekend-2018-Idf (retrieved 11 April 2019). 